\title{
Automatisierte Vermessung der Sichtliniencharakteristik von Bolometerkameras für die Fusionsforschung
}

\author{
Florian Penzel ${ }^{1,2}$, Hans Meister ${ }^{1}$, Till Sehmer ${ }^{1}$, Alexander W. Koch ${ }^{2}$ \\ ${ }^{1}$ Max-Planck-Institut für Plasmaphysik, Boltzmannstr. 2, 85748 Garching \\ ${ }^{2}$ Lehrstuhl für Messsystem- und Sensortechnik, Technische Universität München \\ Tel.: 0893299 1541, e-mail: f.penzel@tum.de
}

\section{Kurzfassung}

Um in einem Fusionsexperiment die Strahlungsleistungsdichte - ein Teil der Energiebilanz - zu bestimmen, wird seit langer Zeit ein Messverfahren auf Grundlage der Metallwiderstands-Bolometer verwendet. Sie messen die emittierte Strahlung des Plasmas breitbandig in einer zeitlichen und lokalen Auflösung und ermöglichen auch die tomografische Rekonstruktion der räumlichen Struktur der Plasmastrahlung.

Für das sich im Aufbau befindliche internationale Fusionsexperiment ITER (International Thermonuclear Experimental Reactor) werden an diese Bolometerkameras besonders hohe Anforderungen gestellt, insbesondere bezüglich der sehr engen Sichtliniencharakteristik und deren präziser Ausrichtung im Plasmagefäß. Für die Entwicklung einer neuen Bolometerkamera wurde ein vollständig automatisierter Prüfstand auf Basis eines KUKA-Roboters für die Vermessung der zweidimensionalen Charakteristik der Sichtlinien (SL) entwickelt.

In diesem Beitrag werden die prinzipielle Funktionsweise dieses Messverfahrens, die erreichbare Messgenauigkeit, insbesondere bezüglich des Einflusses der Kalibrierquelle, sowie die Messreproduzierbarkeit und einige ausgewählte Messergebnisse präsentiert. Außerdem wird das Verfahren für die geometrische Kalibrierung der Komponenten des Messsystems in einem globalen Koordinatensystem, wie z.B. in dem Fusionsexperiment ASDEX (AxialSymmetrisches Divertor-Experiment) Upgrade (AUG), vorgestellt. 


\section{Bolometer für die Fusionsforschung}

Die Funktionsweise eines Metallwiderstands-Bolometers basiert auf der Absorption der einfallenden Strahlungsleistung über ein geeignetes Material und der anschließenden Messung der Temperaturveränderung über ein Widerstandsthermometer [1]. Bild 1 zeigt schematisch die wichtigsten Komponenten einer miniaturisierten Version mit vier Kanälen: Der Detektor (A) wird über ein photolithographisches Verfahren mittels Sputtering und Aufdampfen der unterschiedlichen Schichten hergestellt. Der Widerstand ist durch eine lange mäanderförmige Leiterbahn (B) realisiert, die sich unterhalb des Isolators und des Absorbers befindet. Zwei Detektoren werden zu einer Wheatstonebrücke verschaltet, einer davon ist für die Temperaturkompensation verdeckt. Bildbereich (C) zeigt einen Vier-Kanal-Detektor, (D) als isometrische Ansicht mit dem Gehäuse. Für die Definition der SL ist dem Detektor ein Kollimator (E) vorangebaut.

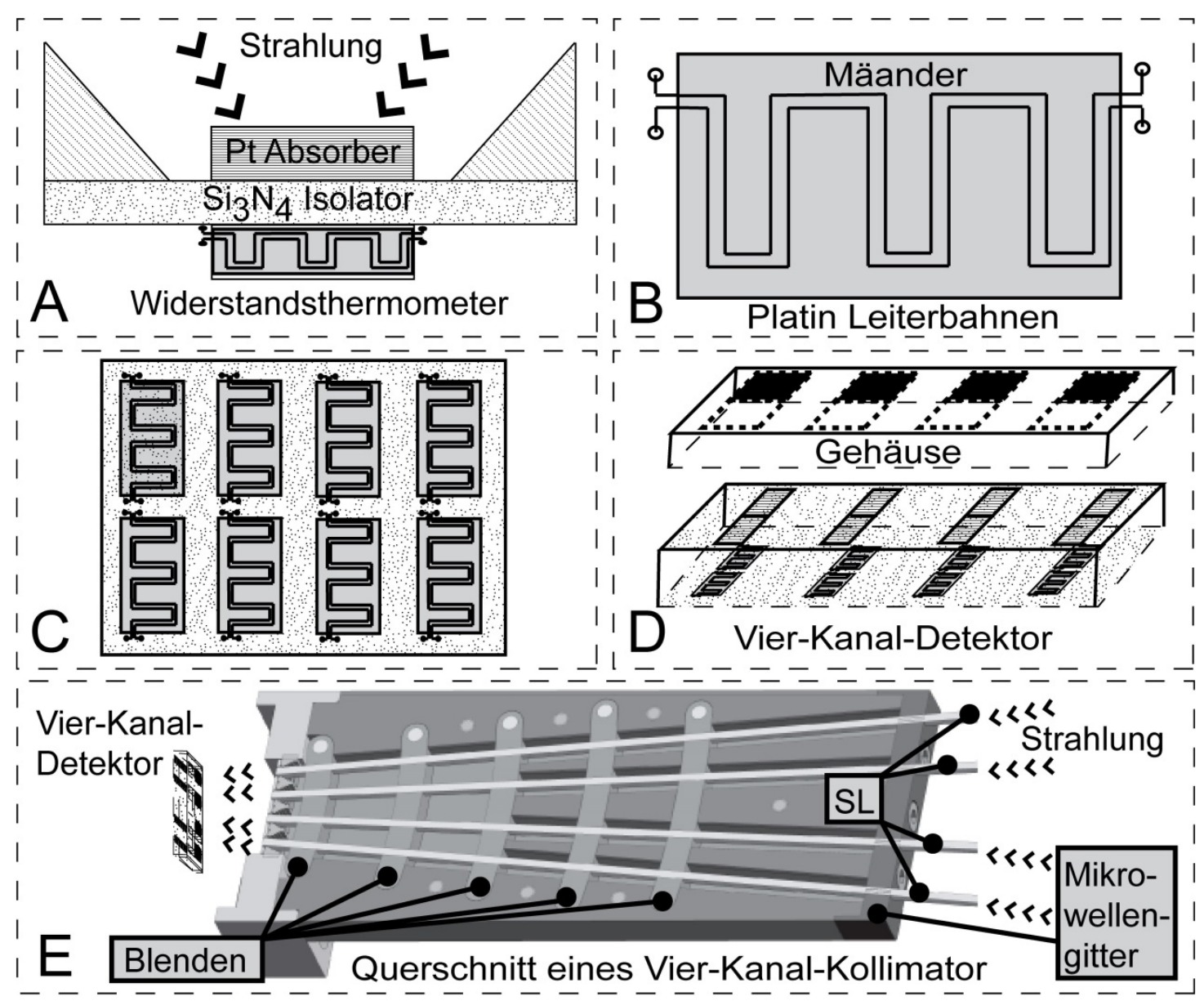

Bild 1: Bolometer Komponenten: Detektor und Kollimator. 
Viele verteilte SL im Experiment ermöglichen die Rekonstruktion des Strahlungsprofils. Dazu ist aber eine genaue Kenntnis der Signalcharakteristik notwendig. Aufgabe des automatisierten Prüfstandes ITER Bolometer Robot Test Rig (IBOROB) ist es, die Charakteristik und die geometrische Orientierung der SL zu vermessen. Im Labor können die Prototypen der für in ITER benötigten Kollimatorkameras damit hinsichtlich Streulicht, Interferenzen, Reflexionen, dem gefordertem Öffnungswinkel und der Konstruktionsqualität bewertet werden [2]. Eingebaut in einem Fusionsexperiment wie AUG kann auch die globale SL-Orientierung des Messsystems bestimmt werden [3].

\section{Automatische Vermessung der Sichtliniencharakteristik}

Bild 2 zeigt den Prüfstand IBOROB im Einsatz in AUG. Ein KUKA Leichtbauroboter richtet eine Strahlungsquelle auf eine Bolometerkamera im Fusionsexperiment oder im Labor aus. Anschließend wird im Bereich der theoretisch erwartenten SL horizontal und vertikal verfahren. Die Position des Roboters, bzw. die Orientierung der Strahlungsquelle, wird mit dem Messsignal des Bolometers korreliert und ermöglicht die Berechnung der Transmission. Als Strahlungsquelle wird ein Laser (Leistung: $100 \mathrm{~mW}$ ) mit einer Wellenlänge von 638nm verwendet, auf dem ein Strahlaufweiter montiert ist, um paralleles Licht mit einem ausreichend großen Strahlungsquerschnitt von $27 \mathrm{~mm}$ zu erzeugen. Die Datenerfassung und Steuerung des Roboters erfolgt ferngesteuert über Netzwerk und über ein eigens programmiertes Softwareinterface in der Programmiersprache LabVIEW ${ }^{\circledR}$.

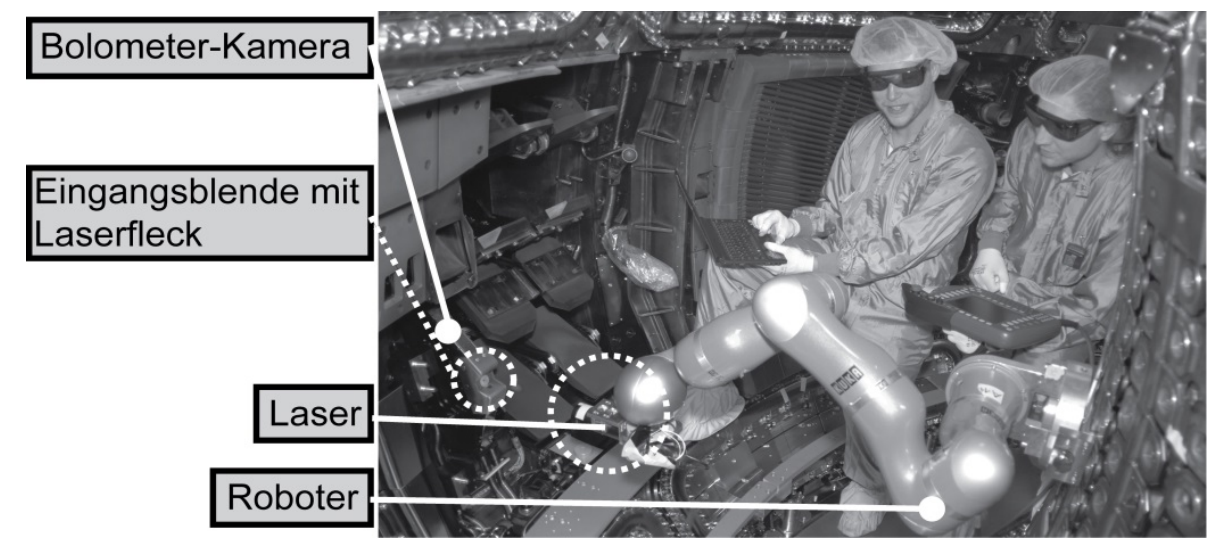

Bild 2: Prüfstand IBOROB im Einsatz in AUG. 
Ein Messergebnis einer Bolometer-Prototypenkamera für ITER (wie in Bild 1(E)) aus einer Labormessung ist in Bild 3 dargestellt. Ein mittlerer Kanal wurde vermessen. Die Transmission ist normiert und in einem dreidimensionalen und Grauskala kodierten Oberflächendiagramm dargestellt. Bei dieser Messung wurde in 0,15 ${ }^{\circ}$ Schritten verfahren. Die Absolutgenauigkeit in dem Laboraufbau beträgt $0,1^{\circ}$. Die vertikale und horizontale Ausdehnung, die Charakteristik und die Form können nun bestimmt werden. Hier ist die SL trapezförmig, der Schwerpunkt und damit die Ausrichtung liegt bei $~ 2,5^{\circ}$ vertikaler und $\sim 0^{\circ}$ horizontaler Richtung im Labor Koordinatensystem (KS). Die Richtung und die Stärke von kleineren Reflexionen und Streulicht wie z.B. bei $4^{\circ}$ vertikaler Orientierung über die ganze horizontale Breite der SL können identifiziert werden. Bei dieser Messung war der Kollimator an der obersten Blende mit einem speziellen Gitter ausgestattet, mit dem Ziel die Mikrowellenstrahlung der Plasmaheizungen bei der Verwendung in ITER zu filtern. Dieses Gitter erzeugt eine regelmäßige Amplitudenmodulation der Transmission, erkennbar an den mehrfachen Maximas des Signals.

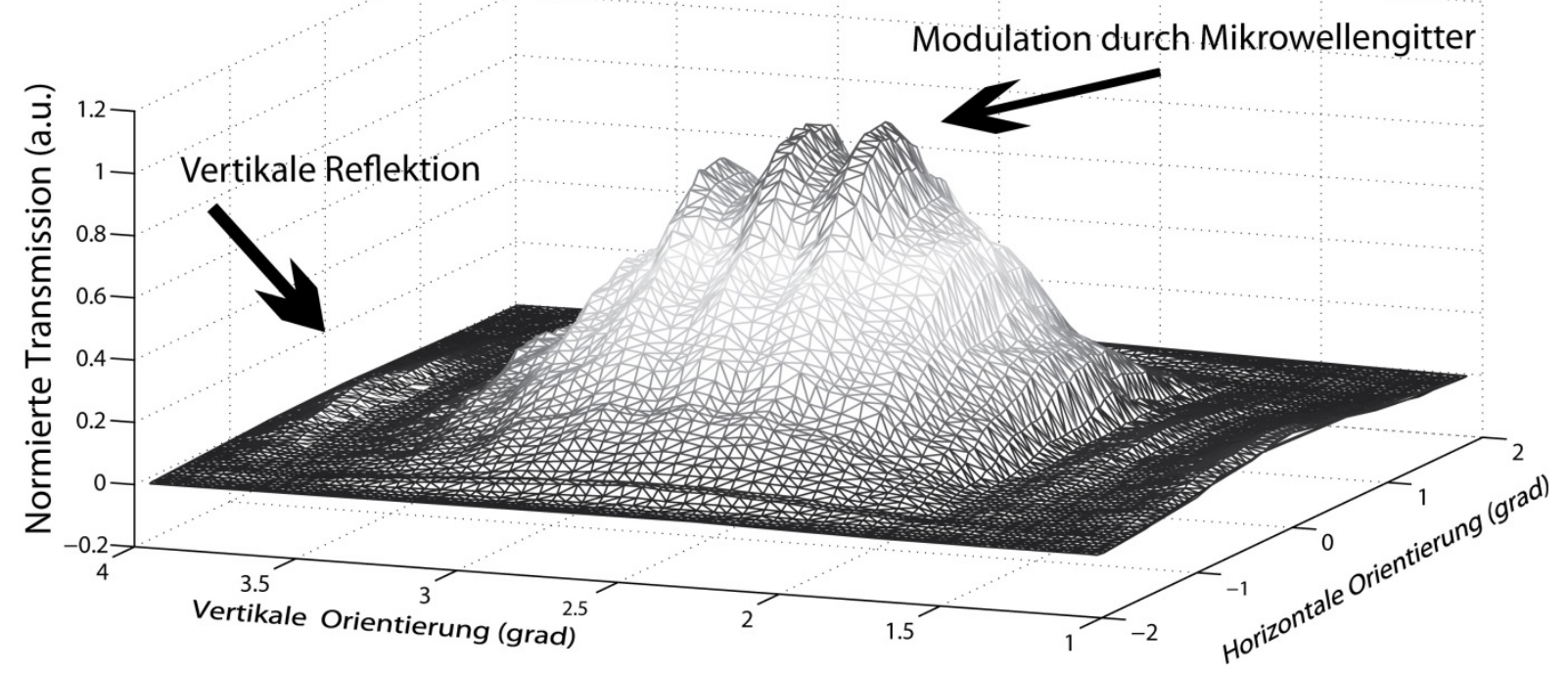

Bild 3: Normierte Transmission einer Bolometersichtlinie aufgetragen über die vertikale und horizontale Orientierung.

Für die Entwicklung einer neuen Bolometerkamera für ITER kann mit verschiedenen Kameraparametern, wie z.B. der Anzahl und Größe der Blenden experimentiert werden, um die SL besser am die Vorgaben anzunäheren. 


\section{Einfluss der Kalibrierquelle auf die Transmission}

Um die Messgenauigkeit, insbesondere auch im Ausblick auf die physikalischen Aussagen bezüglich der Sichtliniencharakteristik und optischer Effekte zu bewerten, wurden unterschiedliche Einflussfaktoren des Prüfstandes untersucht. In diesem Abschnitt wird ein möglicher Einfluss der Kalibrierquelle auf die Transmission diskutiert. Im Prinzip wird davon ausgegangen, dass die Strahlungsquelle vollkommen paralleles Licht auf den Mittelpunkt der Blendenöffnung der Bolometerkamera richtet. Die Wirkung einer unterschiedlichen Fokussierung der Strahlaufweitung wurde durch eine Parametervariation überprüft. In Bild 4 ist die Bolometer-Brückenspannung links über die vertikale Orientierung und rechts über die horizontale Orientierung aufgetragen. Bei dieser Messung wurde die Strahlungsquelle entlang nur einer Achse verfahren (vertikal oder horizontal), da eine 2DMessung für die hier betrachteten Effekte keine zusätzlichen Informationen liefert.

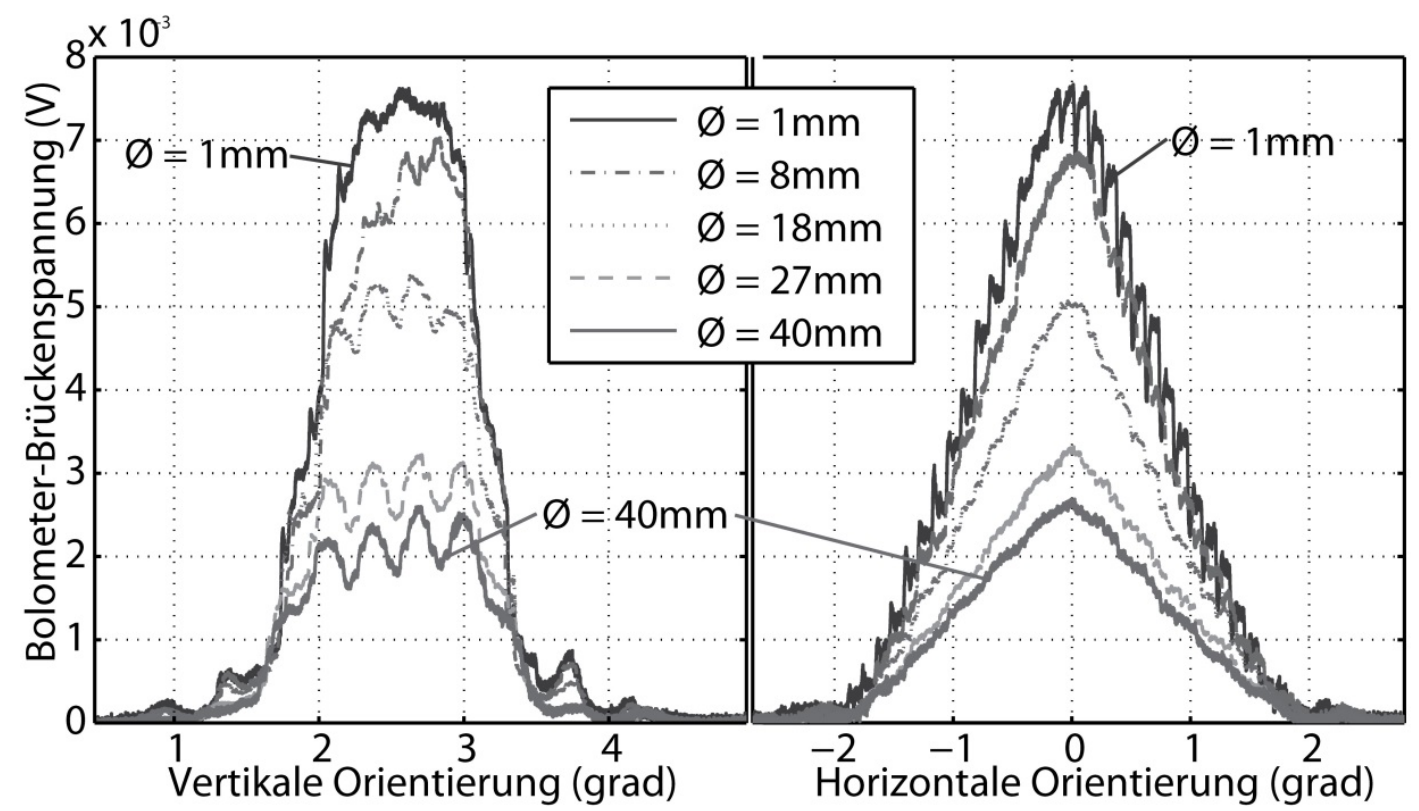

Bild 4: Einfluss der Fokuseinstellung der Strahlungsquelle auf die Transmission.

In der horizontalen Orientierung erzeugt der unterschiedliche Fokus zuerst nur eine Veränderung der Gesamtamplitude des Signals, erst bei der maximalen Fokussierung von $1 \mathrm{~mm}$ lässt sich eine Sägezahnmodulation erkennen, die durch die ungleichmäßige Roboterbewegung erzeugt wird. In der vertikalen 
Orientierung ergibt sich die gleiche Amplitudenveränderung, jedoch verschwindet hier schrittweise die Modulationscharakteristik des Mikrowellengitters mit einem Lochdurchmesser von $\sim 0,5 \mathrm{~mm}$. Ein zu starker Fokus dämpft Signaldetails somit erst ab und lässt diese am Ende ganz verschwinden. Vor der Messung sollte deswegen die Lichtquelle auf ihre Parallelität überprüft werden.

\section{Geometrische Kalibrierung der Kamera in einem globalen Koordinatensystem}

Um Aussagen über die absolute Orientierung des Bolometersystems in einem Fusionsexperiment oder Labor zu treffen, muss die Orientierung von IBOROB vor der Messung bestimmt werden. Hierzu wird der portable Messarm FaroArm $^{\circledR}$ verwendet. In Bild 5 ist ein schematisches Modell von AUG und allen beteiligten KS dargestellt: Drei Kameras (FVC, FHC, FLX), IBOROB (in Sektor 5), das FaroArm ${ }^{\circledR}$ KS und das AUG KS sind eingetragen. Der Messarm ist in einem benachbarten Sektor (6) des Torus montiert.

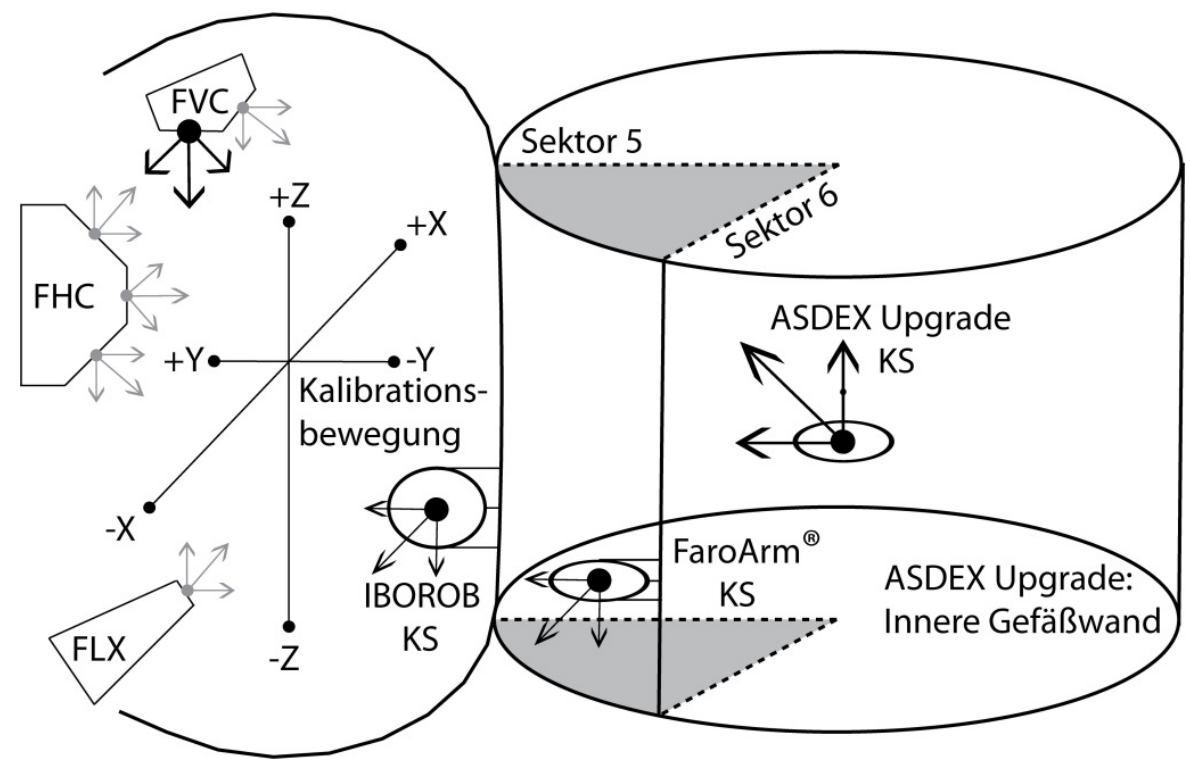

Bild 5: Modell von AUG und allen beteiligten KS.

Der FaroArm ${ }^{\circledR}$ vermisst die Orientierung des Roboters über eine spezielle Kalibrationsbewegung in alle drei Richtungen, der Ursprung des KS lässt sich 
über eine Messung des Roboterfußes konstruieren. Die Bolometerpositionen werden über die Vermessung des Gehäuses und der Eingangsblende bestimmt, die Orientierung des Gefäßes ist über eine Vielzahl von gleichmäßig verteilten Positionsstiften definiert. Das Bolometersystem hat insgesamt 112 Kanäle, Bild 6 zeigt die Vermessung eines Ausschnitt von vier Kanälen der Kamera FVC, die nach unten ausgerichtet ist (schwarz markiertes KS in Bild 5). Zwei unterschiedliche Messungen wurden in den Plot aufgenommen. Messung (1) hat Kanal 79-81 vermessen und Messung (2) Kanal 81-82. Die Kreuze im Plot zeigen die theoretisch erwartete Ausrichtung der Bolometersichtlinien aus CADDaten des Gefäßes. Der vermessene Schwerpunkt lässt sich über eine Integration des Signals berechnen. Es können Unterschiede von $0,5^{\circ}$ bis $1,5^{\circ}$ bezüglich des Erwartungswerts festgestellt werden (vgl. Kanal 82 und Kanal 79). Der größere Abstand zwischen Kanal 80 und 81 ist konstruktionsbedingt.

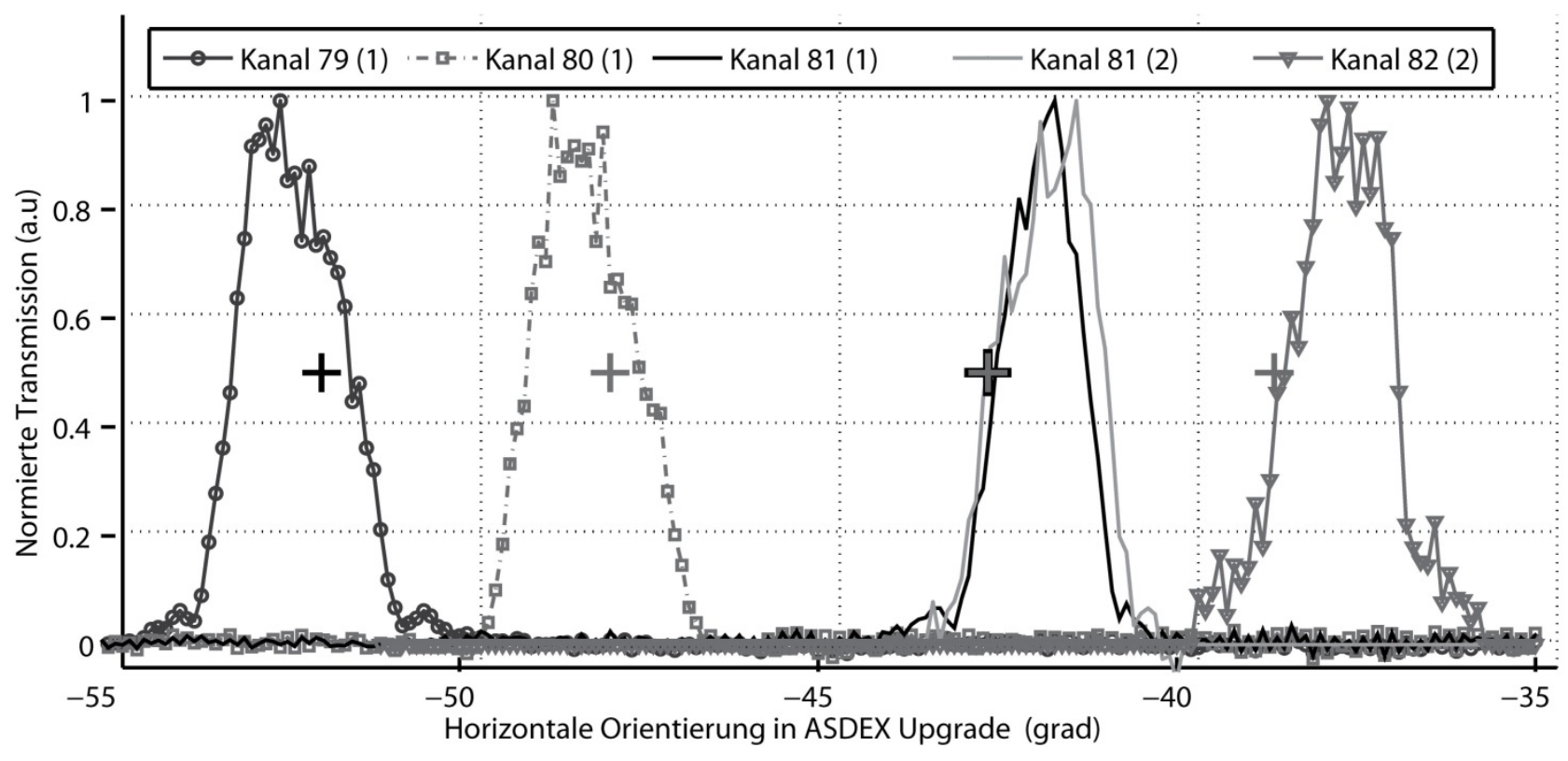

Bild 6: Horizontale Vermessung der FVC Bolometer Kamera.

Außerdem kann durch die zwei unterschiedlichen Messungen die Messgenauigkeit überprüft werden. Die Messungen wurden an zwei unterschiedlichen Tagen durchgeführt und der Roboter wurde dazwischen ausgeschaltet und hatte sich dazu in der Parkposition befunden. Es stellt damit eine Abschätzung der Messungenauigkeit für den Aufbau in AUG im ungünstigsten Fall dar. Bei dem Vergleich von Messung (1) und (2) bei Kanal 81 wurde eine Abweichung des Schwerpunkts von $0,2^{\circ}$ festgestellt. 


\section{$5 \quad$ Fazit und Ausblick}

In diesem Beitrag wurde ein automatisierter Prüfstand zur Vermessung der Charakteristik und Ausrichtung von Bolometersichtlinien für die Fusionsforschung vorgestellt. Es wurde gezeigt, dass die Form der SL zweidimensional mit einer hohen Auflösung vermessen werden kann, Reflexionen durch Streulicht und der Einfluß des Mikrowellengitters können durch die Messung eindeutig identifiziert werden. Der Einflussfaktor der Strahlungsparallelität der Kalibrierquelle auf die Messgenauigkeit wurde diskutiert.

Desweitern wurde gezeigt, dass der Prüfstand zusammen mit einem zusätzlichen Messarm erfolgreich in dem Fusionsexperiment AUG eingesetzt werden kann, um die Orientierung der SL in einem globalen KS zu bestimmen. Die absolute Messungenauigkeit beträgt im Gefäß $0,2^{\circ}$. In der Zukunft könnte das System als Standard-Wartungswerkzeug verwendet werden, um bei Neu- oder Umbauten die SL zu überprüfen. Dies ist ein hoher Aufwand für ein Experiment wie AUG. Für ITER wird ein ähnliches Werkzeug jedoch unabdingbar sein, da SL mit einem sehr schmalen Öffnungswinkel realisiert werden müssen und überprüft werden muss, ob sie von anderen Komponenten des Experimentes abgeschattet oder verdeckt werden.

\section{Literatur}

[1] F. Mast, C. Fuchs, M. Allgäuer, P. Lang, M. Münch, R. Reichle, G. Schramm und G. Weber, "Miniaturisiertes breitbandiges Bolometerarray", Technisches Messen 4, 1997.

[2] F. Penzel, H. Meister, L. Giannone, M. Kannamüller, J. Koll, T. Trautmann and A. W. Koch, "Assessment of line of sight characteristics of ITER bolometer prototype collimators", Fusion Engineering and Design, Proceedings of the 27th Symposium of Fusion Technology (SOFT-27) 88, S. 1267-1270, Jan 2013.

[3] F. Penzel, H. Meister, M. Bernert, T. Sehmer, T. Trautmann, M. Kannamüller , J. Koll and A.W. Koch, „Automated in situ line of sight calibration of ASDEX Upgrade bolometers, Fusion Engineering and Design, Available online 24.April 2014. 